\title{
Image Dehazing using PCA Fusion Technique for Enhanced Road Visibility
}

\author{
Pranali U. Naik \\ Masters of Engineering \\ Industrial Automation \& Radio Frequency \\ Goa College of Engineering \\ Ponda, Goa-India, 403401
}

\author{
Samarth Borkar \\ Asst. Professor \\ Goa College of Engineering \\ Electronics \& Telecommunication Department \\ Ponda, Goa-India,403401
}

\begin{abstract}
Presence of fog and haze significantly reduces the visibility of a scene. Better visibility is crucial for all computer vision applications thus recovering images impaired by haze or dehazing finds its application in the fields of surveillance, tracking, detection and restoration. In this paper fusion based approach using principle component analysis (PCA) technique has been adopted. The novelty of this algorithm is that it does not require any haze depth generation as normally required in many existing methods. Using the original image two images are derived on these images contrast adjustment, and contrast normalization techniques are performed. PCA fusion improves fused image quality and resolution. This method only requires the original image and is simple and easy to implement. As the haze impaired image appears whitish and blurry the details of the road become less evident thus making driving in foggy weather conditions unsafe. Thus, the proposed method concentrates on dehazing for better road visibility. The qualitative and quantitative comparison as compared with existing color fidelity and contrast reveals that our proposed novel method is better at restoring color fidelity and enhancing contrast.
\end{abstract}

\section{General Terms}

Haze, Air light, Contrast Color fidelity.

\section{Keywords}

Principle Component Analysis; Contrast normalization; Dehazing; Haze depth;

\section{INTRODUCTION}

Images captured under fog prevailing conditions inevitably suffer from low contrast and visibility. In hazy weather dust, smoke and other dry particles scatter the light and obscure the clarity of the scene which affects visual recognition and hinders further image processing steps. The light emitted from the scene is mixed with the light coming from all other directions called the airlight [1] which causes a whitish appearance to the captured images. Images also undergo a gradual loss in intensities which is referred to as attenuation. Due to this there is significant decay in the color [2][3].

Computer vision system deals with use of computers for extracting beneficial information from the images. Computer vision finds its usefulness in automatic inspection [4], assisting humans in identification tasks, object recognition, controlling processes, detecting events, navigation the benefits of which could be effectively extracted for medical, military, industrial, traffic surveillance and safety purposes[5].

Computer vision applications depend on the visibility of the images and dehazed images could mislead the functioning of these applications thus dehazing or improving the visibility of degraded image becomes essential [6]. According to the
National Crime Records Bureau (NCRB), in 2014, 9039 road crashes took place as a result of unfavorable weather conditions, especially fog, killing 5,300 people [7]. Since fog degrades the visibility, driving in foggy conditions becomes risky thus to assist the driver camera-based systems have been introduced that provided enhanced vision so that the road edges and vehicles are evident to the driver which ensures safety under different driving conditions.

In this paper fusion based technique [8] is proposed, to overcome the dehazing effect. Fusion refers to combining two input images such that the output image has the maximum information and enhanced visibility[9]. Presence of haze makes the image appear whitish and lowers the contrast, therefore, two images of the original image are obtained by contrast normalization and contrast adjustment. PCA fusion is then performed to fuse the images. Results obtained are tested for foggy road images to test the adequacy of the method for driver assistance system.

\section{LITERATURE REVIEW}

As haze degrades the contrast of the image, contrast enhancement techniques like gamma correction, histogram stretching or equalization or unsharp masking had been implemented [10]. Guo et al [11] proposed a contrastnaturalness-colorfulness assessment system which combines contrast, color naturalness and colorfulness.

In a polarization-based method multiple images with varying brightness levels of the constant scene are derived with different polarization filters and multiple orientations [12,13]. The method could be applied for unmoving scenes, and thin foggy conditions but obtains inaccurate results for intensively foggy scenes. Another shortcoming of this method is that the atmospheric light values need to be manually set and require special equipment like polarizers and not necessarily produce better results.

Dehazing the image using single image is although more difficult. Fast Single Image Haze Removal (FSIHR) [14] can be done using Color Attenuation Prior (CAP) and Multi Scale Fusion (MSF) methods. Tan et al. [15] introduced method based on the fact that foggy images have reduced contrast as compared to clear images. Tan developed a cost function in the framework of Markov random fields based on these two

observations. This method works well for single color or grey foggy images and the disadvantage is that the foggy image could be overly restored as there could be 'Halo effect' and color distortion.

Tarel et.al. [16] have proposed an algorithm for visibility restoration from a single image that is based on a filtering approach and assumes that the depth-map must be smooth except along edges with large depth jump. The approach deals 
with linear operations and requires multiple parameters for adjustment. It is beneficial in terms of its speed and thus could be applied for real-time applications of dehazing. The restored image may be mot good because there are discontinuities in the scene depth and color distortion.

Fattal et al. [17] decomposed a single image in to two components that is light reflected from shading and surface and predicted the scene based on Independent Component Analysis(ICA) assuming that the shading and object depth are locally uncorrelated[18]. The method offers high visibility under inhomogeneous fog or thin foggy conditions but is less effective for images with dense fog or significantly low signal to noise ratio.

He et al. [19] introduced a method based on the observation that some pixels have very low intensity in at least one of the channel and these pixels are knows as dark pixels. Kopf et al. [20] method is relying on the three-dimensional model of the outdoor image. This method does not need multiple images of same scene taken through different degree of polarization. The main drawback of this method is the structure of the real world is significantly varied. This approach is application dependent. Kratz and Nishino [21] proposed a Bayesian defogging algorithm for single image defogging based on the fact that the scene albedo and depth are two statistically independent components. The method offers high visibility and low hallo effect for foggy images but is computationally expensive.

C. O. Ancuti and C. Ancuti [8] proposed a fusion based method. Two images of the same scene are observed by white balancing and contrast enhancement. Luminance, chromaticity and saliency weight maps are computed and fused finally to minimize artifacts introduced by the weight maps; the approach is designed in a multi-scale fashion, using a laplacian pyramid representation of the inputs which is combined with gaussian pyramids of normalized weights.

Most traditional image enhancement methods such as histogram equalization, Retinex, the wavelet transform, usually cannot obtain ideal defogging result. Certain proposed methods [17][22], require transmission map to be estimated accurately and the resultant images produce some halo effects, these methods are found to be invalid when scene object is similar to airlight. Methods based on contrast enhancement [23], produce halo effect and uneven illumination. In order to overcome these problems, fusion based dehazing method using PCA technique is proposed which is less prone to unpleasant artifacts, improves image resolution and hence effectively restores visibility.

\section{PROPOSED METHODOLOGY USING PCA BASED FUSION}

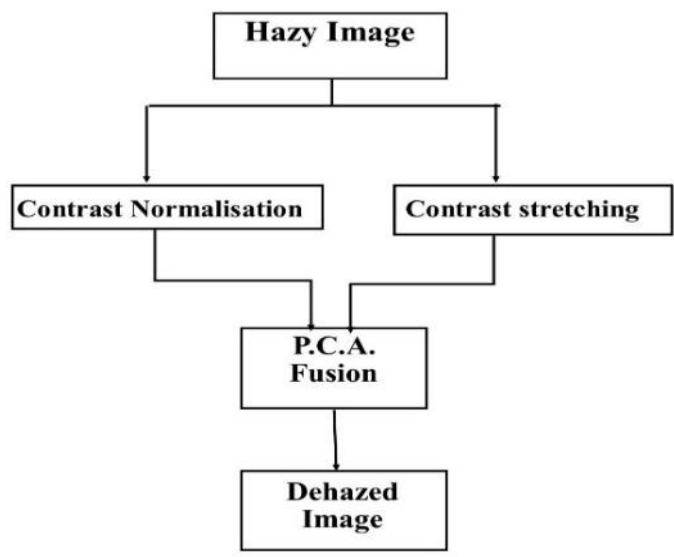

Fig.1: Block diagram for PCA based fusion technique

As seen in Fig.1 from the original hazy image two images are obtained one of which is enhanced using contrast normalization [24] and contrast adjustment is done to the other. The two images are then fused using P.C.A. fusion technique.

The novelty of the algorithm is that the proposed method does not require any haze depth generation as in the existing techniques and is less prone to artifacts.

\subsection{Contrast Normalization}

Contrast normalization or contrast stretching [24] is a method used to normalize the contrast of an image to in order to enhance images with poor contrast. From the input image array maximum and minimum values are determined. The minimum values correspond to the background since the true minimum value is needed the next minimum and maximum values are considered. If both these values are equal image is said to be uniform. Subtracting the minimum value from the maximum value the range of contrast values is obtained,contrast is stretched over these values.

The linear normalization is performed according to the formulae

$$
I_{n}=(I-\operatorname{Min}) \frac{\text { newMax }- \text { newMin }}{\text { Max }- \text { Min }}
$$

Where Min and Max corresponds to minimum and maximum values from the image input array respectively, newMin and newMax corresponds to the next minimum and maximum values from the input array respectively.

Since the desired range is varying from 0 to 255 the minimum value of the intensity range of the image is subtracted from each of the pixel intensity which varies the range from 0 to maximum intensity value. Maximum intensity range is divided by 255 and then each pixel is multiplied by this value.

\subsection{Contrast Adjustment}

Contrast adjustment is used to remaps image intensity values to the full display range of the data type. This technique adjusts the image contrast by scaling the pixel values between upper and lower limits. Pixel values that fall outside the range are saturated to the upper or lower limit value, respectively. 


$$
Y^{\prime}=\left(\frac{\left(Y-\operatorname{low}_{\text {in }}\right)}{\left(\text { high }_{\text {in }}-\text { low }_{\text {in }}\right)}\right)\left(\text { high }_{\text {out }}-\text { low }_{\text {out }}\right)
$$

where $Y^{\prime}$ represent input image pixel under consideration high $_{\text {in, }}$ low $_{\text {in }}$, high $_{\text {out }}$, low $_{\text {out }}$ corresponds to input image high intensity, input image low intensity, output image high intensity and output image low intensity respectively.

\subsection{PCA based Fusion}

The PCA [25] is a mathematical procedure that transforms a number of correlated variables into a number of uncorrelated variables called principal components. Only first few principal components are considered and the rest are discarded which helps in data reduction. The first principal component which shows the most dominant feature accounts for maximum variance in the data and each succeeding component accounts for as much of the remaining variance as possible. The second principal component is made perpendicular of the first. The third principal component is taken in the maximum variance direction in the subspace perpendicular to the first two and so on. Following are the steps involved in PCA algorithm [25]

1.Arrange the two images to be fused in two column vectors.

2.Compute the empirical mean along each column.

3.Calculate the variance-covariance matrix using the formulae.

$$
\begin{aligned}
V_{i} & =\frac{1}{n-1} \sum_{m=o}^{n}\left(X_{i m}-X_{i}\right)^{2} \\
C_{i j} & =\frac{1}{n-1} \sum_{m=1}^{n}\left(X_{i m}-X_{i}\right)\left(X_{j m}-X_{j}\right)
\end{aligned}
$$

where $\boldsymbol{V}_{\boldsymbol{i}}$ is the variance, $\boldsymbol{C}_{\boldsymbol{i}}$ is the covariance of variable $\boldsymbol{i}$ and $\boldsymbol{j}, \boldsymbol{m}$ is the sum over all $\boldsymbol{n}$ objects, $\boldsymbol{X}_{\boldsymbol{i}}$ is the mean of variable $\boldsymbol{i}$, $\boldsymbol{X}_{\boldsymbol{j}}$ is the mean of variable $\boldsymbol{j}, \boldsymbol{X}_{\boldsymbol{i} \boldsymbol{m}}$ is the value of variable $\boldsymbol{i}$ in object $\boldsymbol{m}, \boldsymbol{X}_{\boldsymbol{j} \boldsymbol{m}}$ is the value of variable $\boldsymbol{j}$ in object $\boldsymbol{m}$.

4. The matrix so formed will be a $2 \times 2$ matrix with diagonal elements occupying the variance values and off diagonal elements will have the co-variance value.

5. Compute the eigen values and eigen vectors for the above matrix.

6. Principle components will be the eigen vectors corresponding to higher eigen vectors.

7. Multiple the principle components to the two images then combine the two images.

Principal component analysis is specially used for image compression and image classification and for noise removal [26][27][28], the technique improves the peak signal noise ratio by denoising the image effectively and keeping the data of original image better. The method also improves image resolution.

\section{RESULT}

To prove the effectiveness of our method our results are compared with several existing methods. The results are also tested with road images to test the effectiveness of the method for road traffic surveillance.

Based on the results obtained a quantitative evaluation is performed using the blind measure of Hautière et al. [16] this quality assessment approach deals with computing the ratio between the gradients of the image before and after restoration. This is based on the concept of level of visibility, commonly used in lighting engineering. In, Table 1 . four images (named as ny12, ny17, y01 and y17)are considered where indicator $\boldsymbol{e}$ represents edges newly visible after restoration, indicator $\boldsymbol{r}$ corresponds to the mean ratio of the gradients at visible edges, while indicator $\sum$ represents the percentage of pixels which become completely black or completely white after restoration. To compute this indicator, parameters used in [16] are used. Besides the above mentioned dehazing techniques, the values of these indicators when applying just an unsharp filter are shown in the table 1.

Evaluating the results mentioned in table lit is evident that the values of the descriptor $\sum$ (the percentage of pixels which become completely black or completely white after the restoration) is generally close to zero for the previous methods as well as for our method.

The indicator e corresponds to the effectiveness of the method to restore the edges [23] calculated using Equation (5).

$$
\boldsymbol{e}=\frac{n_{r}-n_{o}}{n_{r}}
$$

where $\boldsymbol{n}_{\boldsymbol{r}}$ and $\boldsymbol{n}_{\boldsymbol{o}}$ correspond to cardinal numbers of the set of visible edges respectively. The results show that for most of the previous methods values corresponding to $\boldsymbol{e}$ are found to be negative whereas only for our method and He et al. [19] of the gradients at visible edges.

The value of $\boldsymbol{r}$ infers the ability of the method to restore the contrast and is calculated using Equation (6).

$$
r=\frac{1}{n_{r}} \sum \log \left(r_{i}\right)
$$

where $\boldsymbol{r}_{i}$ is the ratio of the gradient in the dehazed image and in the original hazy image closely examining restored images obtained from the above-mentioned techniques it is observed that the techniques having low values of indicator $r$ show less spurious edges and artifacts. More over the values of $r$ obtained for our technique are found to be lower in comparison to the other mentioned techniques and thus our method comes out to be less prone to unpleasant artifacts.

For quantitative evaluation comparison between recent dehazing techniques was performed and our technique is shown in Fig. 2. Images restored after performing our technique seem to have increased colour fidelity and contrast and blocky artifacts were less observed as in other techniques.

The proposed algorithm was tested for a set of foggy road images. The compared evaluation is as shown in Fig. 3. The edges of the restored images are prominently evident as compared to edges of the original images. 


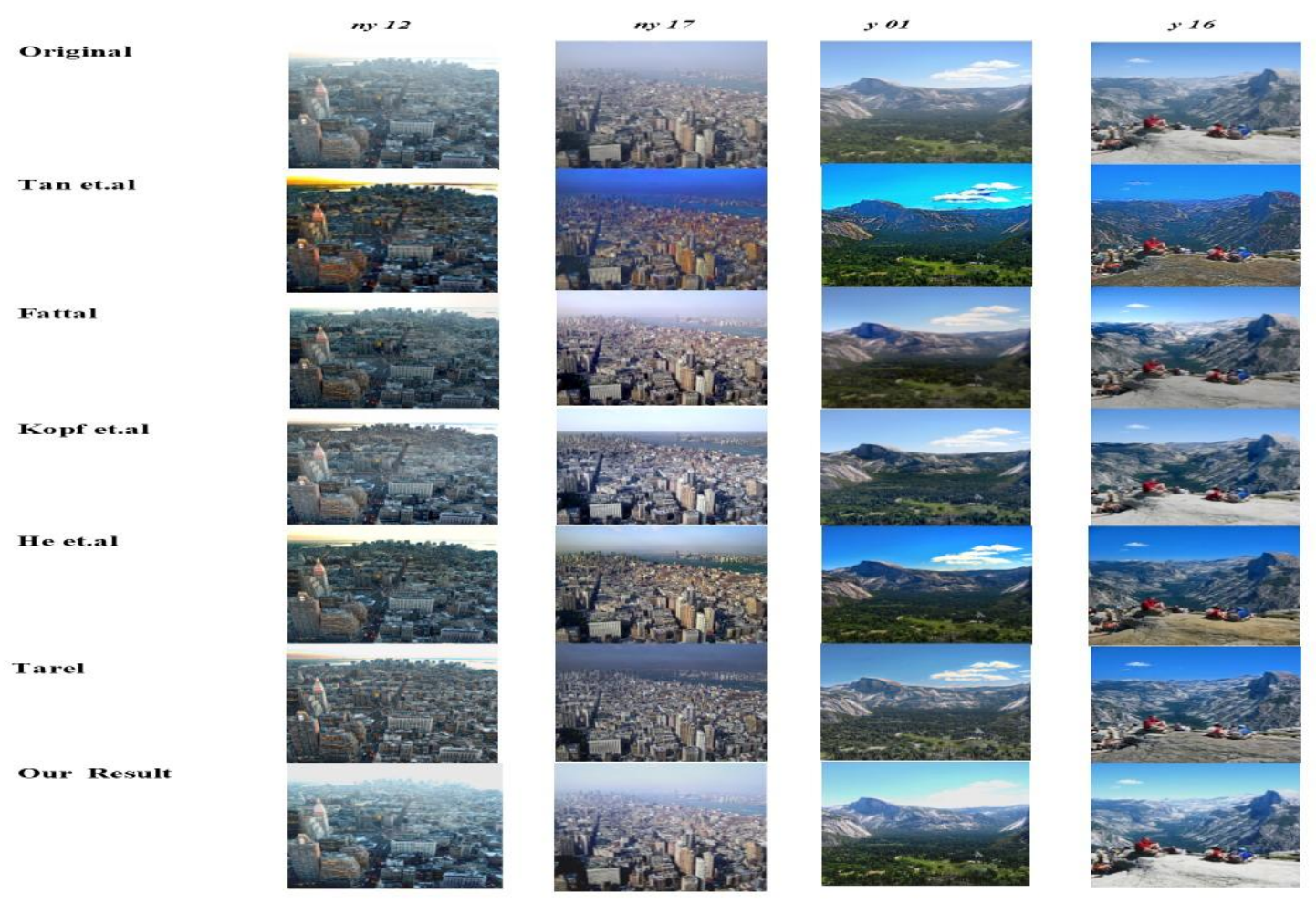

Fig. 2: Comparison of the recent dehazing techniques. Besides the initial hazy images in this figure are displayed the results of Tan [15], Tarel et al. [16], He et al. [19], Fattal [13], Kopf et al. [20] and our technique. Image courtesy (J.-P Tarel database).

Table 1. Quantitative analysis of four images using parameters e, $\sum$ and $r$ of Hautière et al. [16]

\begin{tabular}{|c|c|c|c|c|c|c|c|c|c|c|c|c|c|c|c|c|c|c|c|c|c|}
\hline & \multicolumn{3}{|c|}{ Unshapmasking } & \multicolumn{3}{|c|}{$\operatorname{Tan}[11]$} & \multicolumn{3}{|c|}{ Fattal[13] } & \multicolumn{3}{|c|}{ Kopf [16] } & \multicolumn{3}{|c|}{$\mathrm{He}[15]$} & \multicolumn{3}{|c|}{ Tarel[12] } & \multicolumn{3}{|l|}{ Ours } \\
\hline & $e$ & $\sum$ & $\mathbf{r}$ & $e$ & $\sum$ & $\mathrm{r}$ & $e$ & $e$ & $\sum$ & $\mathbf{r}$ & $e$ & $\sum$ & $\mathrm{r}$ & $e$ & $e$ & $\sum$ & $\mathbf{r}$ & $e$ & $\sum$ & $\mathrm{r}$ & $e$ \\
\hline ny12 & -0.09 & 0.72 & 257 & -0.14 & 0.02 & 234 & -0.06 & -0.09 & 0.72 & 257 & -0.14 & 0.02 & 234 & -0.06 & -0.09 & 0.72 & 257 & -0.14 & 0.02 & 234 & -0.06 \\
\hline ny17 & -0.10 & 1.28 & 2.29 & -0.06 & 0.01 & 222 & -0.12 & -0.10 & 128 & 229 & -0.06 & 0.01 & 222 & -0.12 & -0.10 & 128 & 229 & -0.06 & 0.01 & 222 & -0.12 \\
\hline y01 & 0.04 & 0.27 & 259 & -0.08 & 0.01 & 208 & 0.04 & 0.04 & 027 & 259 & -0.08 & 0.01 & 208 & 0.04 & 0.04 & 0.27 & 259 & -0.08 & 0.01 & 2.08 & 0.04 \\
\hline y16 & 0.09 & 232 & 1.87 & -0.08 & 0.01 & 208 & 0.03 & 0.09 & 232 & 1.87 & -0.08 & 0.01 & 208 & 0.03 & 0.09 & 232 & 1.87 & -0.08 & 0.01 & 208 & 0.03 \\
\hline
\end{tabular}



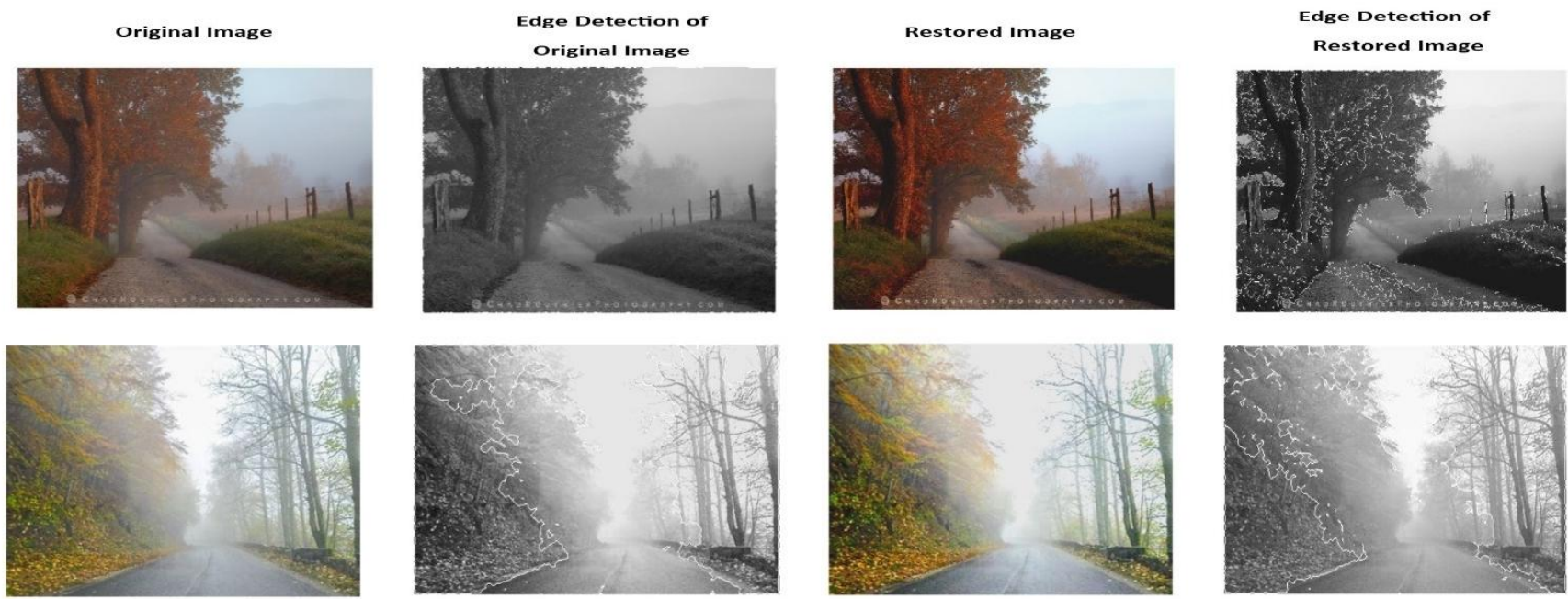

Fig. 3: Comparison between original images and restored images by performing proposed algorithm and their respective edge detection results.

\section{CONCLUSION}

PCA based fusion approach to effectively enhance hazy and foggy images is proposed in this paper. Existing methods are susceptible to unpleasant artifacts and halo effect and no technique is accurate for different kind of circumstances. In the proposed technique two images obtained from the original image are preprocessed by performing contrast normalization on the first image and contrast adjustment on the second. These two images are then fused using PCA fusion technique which improves image resolution. The method has the advantage of enhancing robustly degraded images without estimating the transmission. The quantitative and qualitative comparison shows that the method is found to be less prone to unpleasant artifacts and provides enhanced visibility. The proposed method was also tested for road edge detection to test its effectiveness for road surveillance application. The road edges are found to be better visible post restoration. Although the method yields better visibility and edge restoration in comparison to previous techniques it is found to be less effective as the visibility distance increase in future implementation of dehazing of mosaic images with continuous tone haze removal is intended.

\section{REFERENCES}

[1] S. P. Patel and M. Nakrani, "A review on methods of image dehazing," International Journal of Computer Applications (0975 - 8887) Volume 133 - No.12, January 2016.

[2] W.Wang and X.Yaun, "Recent advances in image dehazing", IEEE/CAA Journal of Automatica Sinica, Vol-4, 2017.

[3] C. Chengtao, Z. Qiuyu and L. Yanhua, "A survey of image dehazing approaches" IEEE/CCDC, 23-25 May 2015.

[4] B.A. Baumann, M. Boltz, J. Ebling, M. Koenig, H.S. Loos, M. Merkel, W. Neim, J. K. Warzelhan and J. Yu "A review and comparison of measures for automatic video surveillance systems," EURASIP Journal on Image and Video Processing 2008, 30 (2008).
[5] E Kermani, D Asemani, "A robust adaptive algorithm of moving object detection for video surveillance", EURASIP J. Image Video Process. 2014(27), 1-9 (2014)

[6] D. Neelima and G. Mamidisetti, "A computer vision model for vehicle detection in traffic surveillance," International Jjournal of Science and Advance Technology Volume-2, Issue-5, 1203 - 1209.

[7] C. O. Ancuti, C. Ancuti, and P. Bekaert, "Effective single image dehazing by fusion," in Proc. 17th IEEE Int. Conf. Image Process., Hong Kong, China, 2010, pp. 35413544 .

[8] Y. Yang, D.S. Park, S. H., and N. Rao, " Medical image fusion via an effective wavelet-based approach", EURASIP Journal on Advances in Signal Processing Volume 2010.

[9] K.Kim, “Contrast enhancement system using spatially adaptive histogram equalization with temporal filtering", in IEEE Trans.Consum.Electron, 1998.

[10] Z. Guo, "Objective assessment method for the clearness effect of image defogging algorithm", Acta Automatica Sinica, 201239(9):1410-1419.

[11] Y.Y. Schechner, S.G. Narasimhan and S.K. Nayar, "Instant Dehazing Of Images Using Polarization", Proc. Computer Vision \& Pattern Recognition Vol. 1(2001).

[12] A. S. Narasimhan and S. Nayar, "Contrast restoration of weather degraded images", IEEE Trans. Pattern Analysis and Machine Intelligence, vol. 25, no. 6, June 2003.

[13] K. Katiyar and N.Verma, "Single image haze removal algorithm using color attenuation prior and multi-Scale fusion", International Journal of Computer Applications (0975 - 8887) Volume 141 - No.10, May 2016.

[14] R. T. Tan, "Visibility in bad weather from a single image", in IEEE Conf. on Computer Vision and Pattern Recognition, 2008.

[15] J.-P. Tarel and N. Hautiere, "Fast visibility restoration from a single color or gray level image," in Proc. IEEE Int. Conf. Comput. Vis., Sep.-Oct. 2009, pp. 2201-2208. 
[16] R. Fattal, "Single image dehazing," ACM Trans. Graph., vol. 27, no. 3, p. 72, Aug. 2008.

[17] L. Kratz and K. Nishino, "Factorizing scene albedo and depth from a single foggy image", in Proc. IEEE Int. Conf. Comput. Vis., Sep.-Oct. 2009, pp. 1701-1708.

[18] K. He, J. Sun, and X. Tang, "Single image haze removal using dark channel prior", IEEE Transactions on Pattern Analysis and Machine Intelligence, Vol. 33, No. 12, December 2011.

[19] J. Kopf, B. Neubert, B. Chen, M. Cohen, D. Cohen-Or, O. Deussen, M. Uyttendaele, and D. Lischinski, "Deep photo: model-based photograph viewing," ACM Trans. Graph., vol. 27, no. 5, p. 116, 2008.

[20] L. Kratz and K. Nishino, "Factorizing scene albedo and depth from a single foggy image", in Proc. IEEE Int. Conf. Comput. Vis., Sep.-Oct. 2009, pp. 1701-1708.

[21] K. He, J. Sun, and X. Tang, "Single image haze removal using dark channel prior", In IEEE CVPR, 2009.

[22] N. Hautiere, J.-P. Tarel, D. Aubert, and E. Dumont, "Blind contrast enhancement assessment by gradient ratioing at visible edges", J. Image Anal. Stereo, vol. 27, pp. 87-95, 2008.

[23] K. Iqbal, M. Odetayo, A. James, R. A enhancement and. Salam and A. Z. Talib,"Enhancing the low quality images using unsupervised color correction methods", in IEEE International Conference on Systems Man and Cybernetics, Istanbul, 2010.

[24] V. Gupta and A.V. Band, "Adaptive improved PCA with wavelet transform for image denoising", IJCA (0975 8887) Volume 82, November 2013.

[25] S. Pal and R.Mahakud , M. Sahoo, "PCA based Image Denoising using local pixel grouping", IJCA Special Issue on "2nd National Conference- Computing, Communication and Sensor Network" CCSN, 2011.

[26] V.P.S. Naidu and J.R. Rao, "Pixel level fsion using pribciple component analysis" Defence Science Journal, Vol. 58, May 2008, pp. 338-352,2008.

[27] R.P. Desale and S. V. Verma "Study and analysis of PCA, DCT \& DWT based image fusion techniques”ICSRP-7-8 Feb. 2013.

[28] 